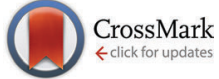

Cite this: Phys. Chem. Chem. Phys. 2014, 16, 20741

Received 14th May 2014, Accepted 11th August 2014

DOI: $10.1039 / c 4 c p 02091 j$

www.rsc.org/pccp

\title{
Optimization of thermoelectric efficiency in SnTe: the case for the light band $\dagger$
}

\author{
Min Zhou, $^{\mathrm{ab}}$ Zachary M. Gibbs, $\ddagger^{\mathrm{c}}$ Heng Wang, ${ }^{\mathrm{b}}$ Yemao Han, ${ }^{\mathrm{a}}$ Caini Xin, ${ }^{\mathrm{a}}$ \\ Laifeng Li*a and G. Jeffrey Snyder*bd
}

\begin{abstract}
$\mathrm{p}$-Type $\mathrm{PbTe}$ is an outstanding high temperature thermoelectric material with zT of 2 at high temperatures due to its complex band structure which leads to high valley degeneracy. Lead-free SnTe has a similar electronic band structure, which suggests that it may also be a good thermoelectric material. However, stoichiometric SnTe is a strongly p-type semiconductor with a carrier concentration of about $1 \times 10^{20} \mathrm{~cm}^{-3}$, which corresponds to a minimum Seebeck coefficient and $z T$. While in the case of $\mathrm{p}$-PbTe (and n-type $\mathrm{La}_{3} \mathrm{Te}_{4}$ ) one would normally achieve higher zT by using high carrier density in order to populate the secondary band with higher valley degeneracy, SnTe behaves differently. It has a very light, upper valence band which is shown in this work to provide higher zT than doping towards the heavier second band. Therefore, decreasing the hole concentration to maximize the performance of the light band results in higher zT than doping into the high degeneracy heavy band. Here we tune the electrical transport properties of SnTe by decreasing the carrier concentration with iodine doping, and increasing the carrier concentration with Gd doping or by making the samples Te deficient. A peak zT value of 0.6 at $700 \mathrm{~K}$ was obtained for $\mathrm{SnTe}_{0.985} \mathrm{I}_{0.015}$ which optimizes the light, upper valence band, which is about $50 \%$ higher than the other peak zT value of 0.4 for $\mathrm{Gd}_{z} \mathrm{Sn}_{1-z \mathrm{~T}} \mathrm{e}$ and $\mathrm{SnTe}_{1+y}$ which utilize the high valley degeneracy secondary valence band.
\end{abstract}

\section{Introduction}

Supplying and maintaining a clean energy supply is an increasingly important goal. Waste heat recovery using thermoelectric materials is one pathway towards this end, but conversion efficiency in these materials is still quite low. The figure of merit, $\mathrm{zT}=\alpha^{2} T / \rho\left(\kappa_{\mathrm{e}}+\kappa_{\mathrm{L}}\right)$, determines the conversion efficiency where $\alpha$ is the Seebeck coefficient, $T$ is the temperature, $\rho$ is the electronic resistivity, and $\kappa_{\mathrm{e}}$ and $\kappa_{\mathrm{L}}$ are, respectively, electronic and lattice contributions to thermal conductivity. Lead chalcogenides, which have the rock salt structure, are some of the most studied thermoelectric materials and have a record high figure of merit (zT) between 1.4 and 2.2. ${ }^{1-3}$ One mechanism of p-type PbTe's outstanding thermoelectric performance is thought to be due to its complex valence

\footnotetext{
${ }^{a}$ Key Laboratory of Cryogenics, Technical Institute of Physics and Chemistry, Chinese Academy of Sciences, Beijing 100190, China.

E-mail: laifengli@mail.ipc.ac.cn

${ }^{b}$ Materials Science, California Institute of Technology, 1200 California Blvd., Pasadena, CA 91125, USA. E-mail: jsnyder@caltech.edu

${ }^{c}$ Division of Chemistry and Chemical Engineering, California Institute of Technology, 1200 E. California Blvd., Pasadena, CA 91125, USA

${ }^{d}$ ITMO University, Saint Petersburg, Russia

$\dagger$ Electronic supplementary information (ESI) available. See DOI: 10.1039/ c4cp02091j

\$ Min Zhou and Zachary M. Gibbs have contributed equally to this work.
}

band structure, especially at high temperatures where the energy of primary and secondary maximums are thought to be aligned-leading to extraordinarily high valley degeneracy. ${ }^{4}$

In SnTe, one might also expect good thermoelectric performance because it shares many of the same characteristics with PbTe; specifically, both exist in the rock salt crystal structure and both have multiple valence bands ${ }^{5}$ which contribute to the thermoelectric properties. However, unlike PbTe, SnTe is inherently riddled with defects which results in a heavily doped $\left(p \sim 10^{20}-10^{21} \mathrm{~cm}^{-3}\right.$ ) material and a mediocre zT (around 0.5 at $900 \mathrm{~K}) .{ }^{6,7}$ Nonetheless, several studies have confirmed the existence of two valence bands (as in PbTe) and have estimated their transport parameters. ${ }^{6,8-11}$

SnTe has a large valence band offset, $\Delta E$, of around $0.3-0.4 \mathrm{eV}$ at room temperature-larger than PbTe which is closer to $0.1 \mathrm{eV}$ (Fig. 1c). ${ }^{9,12}$ The two valence bands in SnTe are known to give rise to a unique Seebeck coefficient behavior as the carrier concentration is varied (Seebeck Pisarenko relation). A minimum Seebeck coefficient is observed in the Pisarenko plot (Fig. 1a) near $n=1-2 \times 10^{20} \mathrm{~cm}^{-3}$, followed by a local maximum at about $n=8 \times 10^{20} \mathrm{~cm}^{-3}$. Theoretical calculations confirm the existence of two near-edge valence bands and their positions in $k$-space and have provided some insights into their character. ${ }^{13-16}$ Very recently Zhang et al. reported an enhanced Seebeck coefficient in SnTe doped with In. An improved maximum zT 

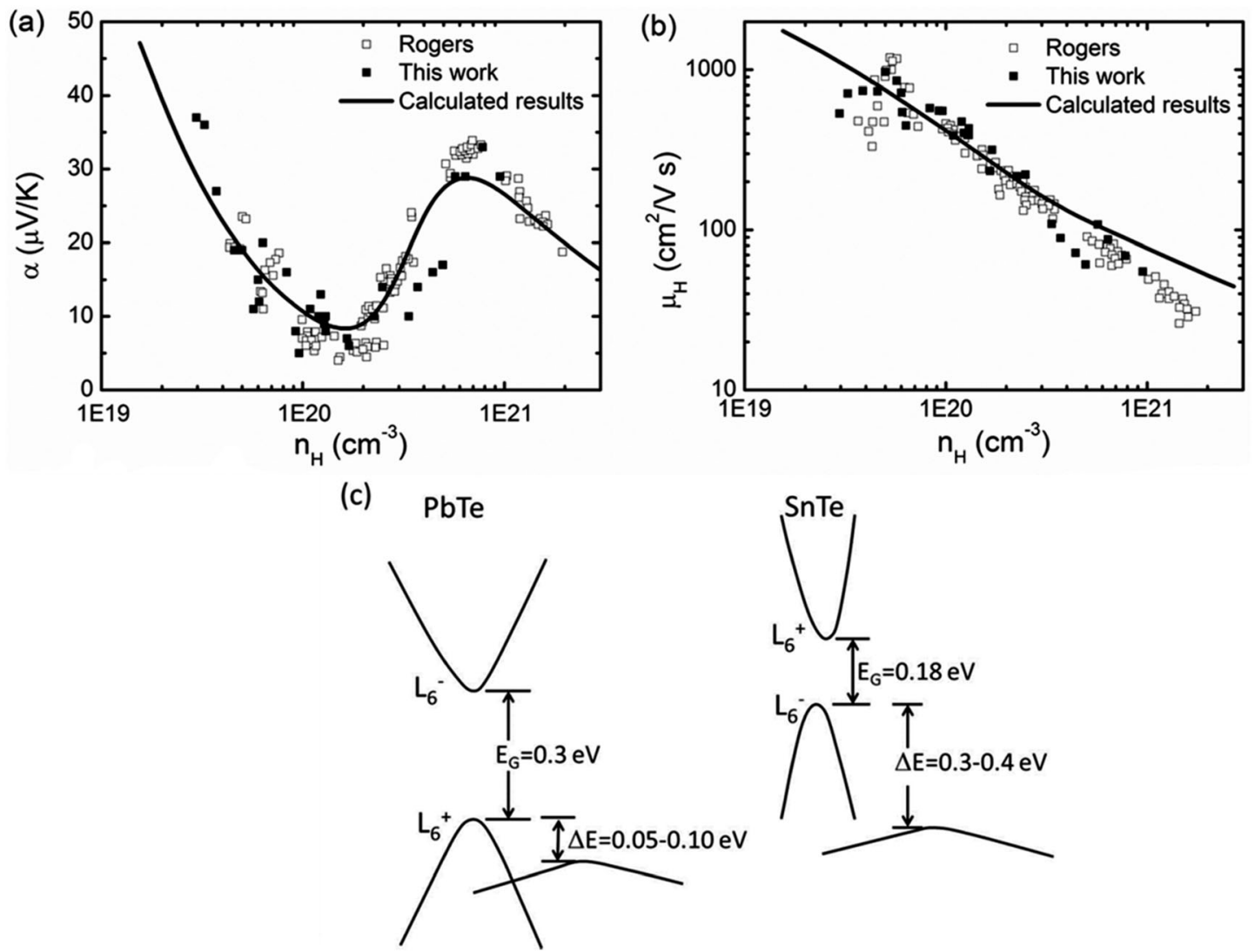

Fig. 1 (a) Seebeck coefficient and (b) Hall mobility as a function of Hall carrier concentration at $300 \mathrm{~K}$ for $\mathrm{SnTe}_{1-x} \mathrm{I}_{x}, \mathrm{SnTe}_{1+y}$, and Gd $\mathrm{Sn}_{1-z} \mathrm{Te}_{\mathrm{S}} \mathrm{Solid}$ squares are our experimental results, open squares are Rogers' reported results, ${ }^{9}$ solid curves are calculated from a two-band model. (c) A schematic diagram of the near edge band structure in PbTe and SnTe. (Rogers et al. reported a band offset of $0.3 \mathrm{eV}$ for SnTe. Our results yielded different fitting parameters, and we found that $0.4 \mathrm{eV}$ was required for the best fit.)

value of 1.1 was observed at $873 \mathrm{~K},{ }^{17}$ suggesting that SnTe may in fact be a promising thermoelectric material. Tan reported a high zT of 1.3 for Cd-doped SnTe with endotaxial CdS nanoscale precipitates. ${ }^{18}$ Han and Chen et al. reported $z \mathrm{~T}$ of $0.9-1$ for SnTe-AgSbTe ${ }_{2}$ alloys. ${ }^{19,20}$ Other than thermoelectric properties, other studies have discussed SnTe and its alloys as useful for long wavelength detectors, ${ }^{21-23}$ or most recently as topological insulators. ${ }^{14,24,25}$

The unique Seebeck coefficient behavior of SnTe as the carrier concentration stimulates our interest to explore the nature of electrical transport in SnTe and optimize the thermoelectric properties. Finding the optimum doping level in semiconductors with complicated band structures is crucial to obtain a thermoelectric material with the optimum performance. In this work, we will show that both the Seebeck coefficient and zT value increase by substituting either donor (I) or acceptor (extra Te and Gd) dopants. We observe that the peak zT value $\left(\mathrm{zT}_{\max , 1}=0.6\right.$ at $\left.673 \mathrm{~K}\right)$ of I-doped SnTe with a decreased carrier concentration $\left(3 \times 10^{19} \mathrm{~cm}^{-3}\right)$ is higher than the other peak $\mathrm{zT}$ value of extra Te or Gd-doped SnTe $\left(\mathrm{zT}_{\max , 2}=0.4\right.$ at $\left.773 \mathrm{~K}\right)$ with an increased carrier concentration $\left(6 \times 10^{20} \mathrm{~cm}^{-3}\right)$, suggesting that the light, primary valence band is most important in these systems. This unique behavior is contrary to the behavior in the lead chalcogenides where the second, heavy band usually leads to an improved figure of merit.

\section{Experimental}

Polycrystalline samples of $\operatorname{SnTe}_{1-x} \mathrm{I}_{x}(0 \leq x \leq 0.02), \mathrm{SnTe}_{1+y}$ $(0<y \leq 0.015)$, and $\mathrm{Gd}_{z} \mathrm{Sn}_{1-z} \mathrm{Te}(0<z \leq 0.02)$ were prepared using a melt alloying and hot pressing technique. Pure elements and $\mathrm{TeI}_{4}$ (Sn, 99.999\%; Te, 99.999\%; Gd, 99.99\%; $\mathrm{TeI}_{4}$, 99.999\%, ultra dry) were weighed out according to each composition and loaded into quartz ampoules, which were then evacuated and sealed. The sealed ampoules were slowly heated to $1273 \mathrm{~K}$ and kept for $24 \mathrm{~h}$ followed by water quenching. The ingots obtained were further annealed at $973 \mathrm{~K}$ for $120 \mathrm{~h}$ before being crushed and ground into fine powders. The powders were then hot pressed at $823 \mathrm{~K}$ under $1 \mathrm{~atm}$ argon with $40 \mathrm{MPa}$ pressure for $30 \mathrm{~min}$. A typical disk shaped sample is obtained which is $12 \mathrm{~mm}$ in diameter with density no less than $95 \%$ of theoretical density $\left(6.46 \mathrm{~g} \mathrm{~cm}^{-3}\right)$. The electrical resistivities and Hall coefficients $\left(R_{\mathrm{H}}\right)$ were measured by using the van der Pauw method in a magnetic field up to $\pm 2 \mathrm{~T}^{26}$ The Seebeck coefficients were obtained by measuring the thermoelectric voltages as well as 
temperatures with T-type thermocouples. ${ }^{27}$ The thermal conductivities were obtained by $\kappa=C_{\mathrm{p}} \lambda d$ with the thermal diffusivity $\lambda$ measured by the laser flash method (Netzsch LFA 457), where $d$ is the geometric density. The heat capacity $C_{\mathrm{p}}$ was determined by $C_{\mathrm{p}}=C_{\mathrm{p}, 300}+C_{\mathrm{p} 1} \times\left((T / 300)^{\alpha}-1\right) /\left((T / 300)^{\alpha}+C_{\mathrm{p} 1} / C_{\mathrm{p}, 300}\right),{ }^{28}$ where $T$ is the absolute temperature and $C_{\mathrm{p}, 300}$ is the specific heat capacity at $300 \mathrm{~K}$. For SnTe, $C_{\mathrm{p}, 300}$ is $0.1973 \mathrm{~J} \mathrm{~g}^{-1} \mathrm{~K}^{-1}, C_{\mathrm{p} 1}$ is $0.115 \mathrm{~J} \mathrm{~g}^{-1} \mathrm{~K}^{-1}$, and $\alpha$ is $0.63 .^{28}$ All the test data were collected during both heating and cooling with both datasets shown.

Transport properties were modeled following previous work. $^{29,30}$ The light and heavy valence band properties were calculated by evaluating the full generalized Fermi integrals as a function of chemical potential. The light band was assumed to be a nonparabolic, Kane band, with a nonparabolicity parameter, $\beta$, given by $\kappa_{\mathrm{B}} T / E_{\mathrm{g}}$, where $E_{\mathrm{g}}$ was assumed to be constant at $0.18 \mathrm{eV},{ }^{31}$ while the heavy band was modeled as a parabolic band. (More information about the specific modeling parameters can be found in the ESI. $\dagger$ )

\section{Results and discussion}

The measured Hall carrier concentrations $\left(p_{\mathrm{H}}=1 / e R_{\mathrm{H}}\right)$ of $\mathrm{SnTe}_{1-x} \mathrm{I}_{x}, \mathrm{SnTe}_{1+y}$ and $\mathrm{Gd}_{z} \mathrm{Sn}_{1-z}$ Te samples at $300 \mathrm{~K}$ are shown in Fig. 2a. The Hall carrier concentration of stoichiometric SnTe was found to be around $1.1 \pm 0.2 \times 10^{20} \mathrm{~cm}^{-3}$ at $300 \mathrm{~K}$ when prepared by using the described method. This value is slightly lower than Zhang's report (about $2 \times 10^{20} \mathrm{~cm}^{-3}$ ). ${ }^{17}$ Extra Te is thought to induce cation vacancies which act as double acceptors. ${ }^{4,32}$ Brebrick, whose data are also shown in Fig. 2, closely studied Te solubility in SnTe and concluded that the phase width always leaned towards the Te-rich side due to Sn vacancies that lead to the heavily p-type character of intrinsic SnTe. Our work agrees with the cation vacancy mechanism and yields $\sim 1.7$ holes per Te atom, although, Brebrick saw more ( 3 holes per Te atom). ${ }^{6}$ Because Brebrick used carefully controlled and measured data for Te content, while we use nominal composition only, Te loss through vaporization during synthesis is a plausible explanation for the difference. Dopant solubility in SnTe has been thoroughly studied by Rogacheva et al., they investigated the complexities involved with doping phases which are intrinsically nonstoichiometric. ${ }^{33}$

Gd with normal valence $\mathrm{Gd}^{3+}$ might be expected to substitute for $\mathrm{Sn}^{2+}$ and be an electron donor, but instead Gd is observed to cause an increase in the p-type, hole carrier concentration. Similar results were reported by Story et al. who suggest that Gd is a resonant dopant. However, we observed no Seebeck increase relative to Te-doped samples in this work which would indicate resonant states-probably due to a lower Gd content $(<1 \%)$ and higher temperatures in comparison to the literature. ${ }^{34}$ While the exact mechanism of Gd doping is not clear, the Gd-doped samples showed a linear increase in $n_{\mathrm{H}}$ with Gd doping of $z>0.0025$.

We also attempted to counterdope SnTe by substituting Te with iodine. While much work has been done on cationic substitutions, far fewer studies study how SnTe is affected by anion substitutions. As pointed out by Rogacheva et al., the cationic dopants that have the highest solubility are those which have similar ionic radii to $\mathrm{Sn}$. Hence, iodine should be a good candidate for anionic substitutional doping in SnTe. As observed in Fig. 2a, the carrier concentration linearly decreased to as low as $3 \times 10^{19} \mathrm{~cm}^{-3}$ with $\sim 40 \%$ doping efficiency assuming that one electron is donated per iodine atom. The doping efficiency appears to be linear until $N_{\mathrm{I}} \sim 25 \times 10^{19} \mathrm{~cm}^{-3}(x=0.015)$ where the carrier concentration continues to decrease, but at a slower rate. The observed carrier concentration has been achieved by previous authors, ${ }^{35-37}$ but their interpretation of it and its significance to the thermoelectric properties was not thoroughly studied.

The measured temperature dependent transport data for samples with nominal composition $\mathrm{SnTe}_{1-x} \mathrm{I}_{x}, \mathrm{Gd}_{z} \mathrm{Sn}_{1-z} \mathrm{Te}$, and $\mathrm{SnTe}_{1+y}$ are shown in Fig. 3. Stoichiometric SnTe data reported by Zhang et al. (green dashed lines for samples with a slightly different $n_{\mathrm{H}}$ than SnTe in this work) are also shown. Degenerate semiconducting behavior, indicated by increasing Seebeck coefficient and resistivity with temperature, is observed for all samples. As the iodine content is increased, the measured Seebeck coefficient and electrical resistivity increase, consistent
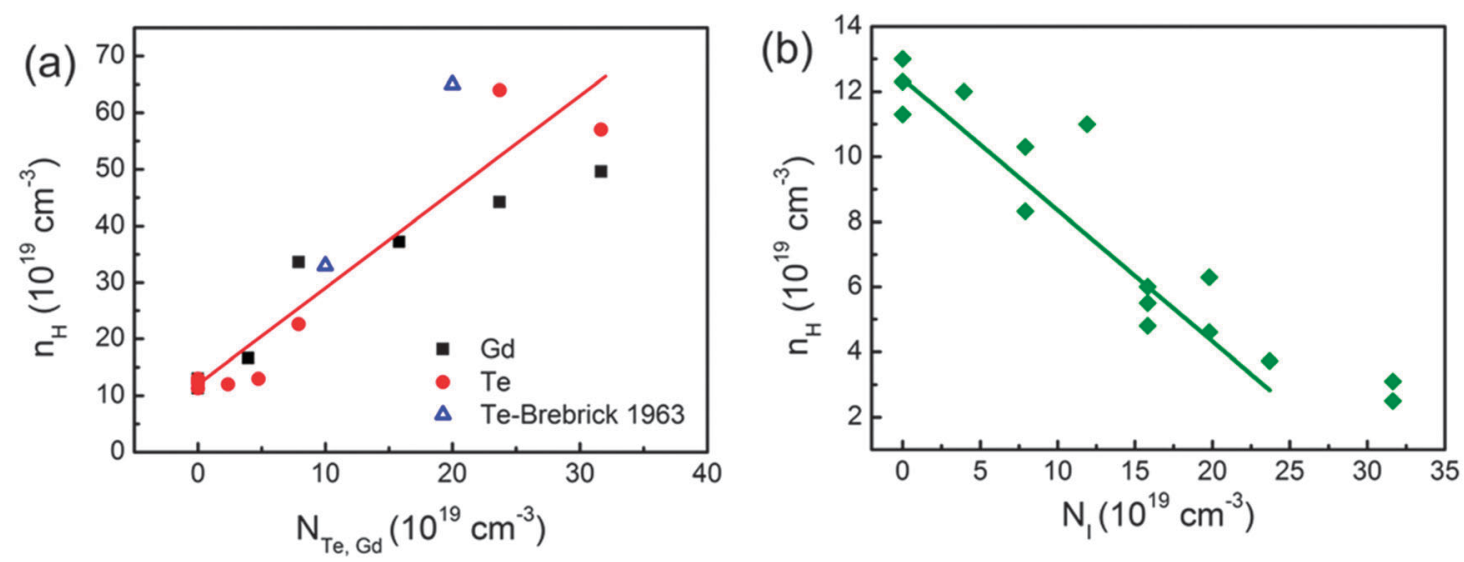

Fig. 2 Hall carrier concentration as a function of dopant concentration (a) Gd and Te excess as acceptors, (b) I as a donor. Solid lines are guide to the eye for our data and correspond to 1.7 holes per atom for the Gd/excess Te case shown in a, and 0.4 electrons per iodine atom as in b. 

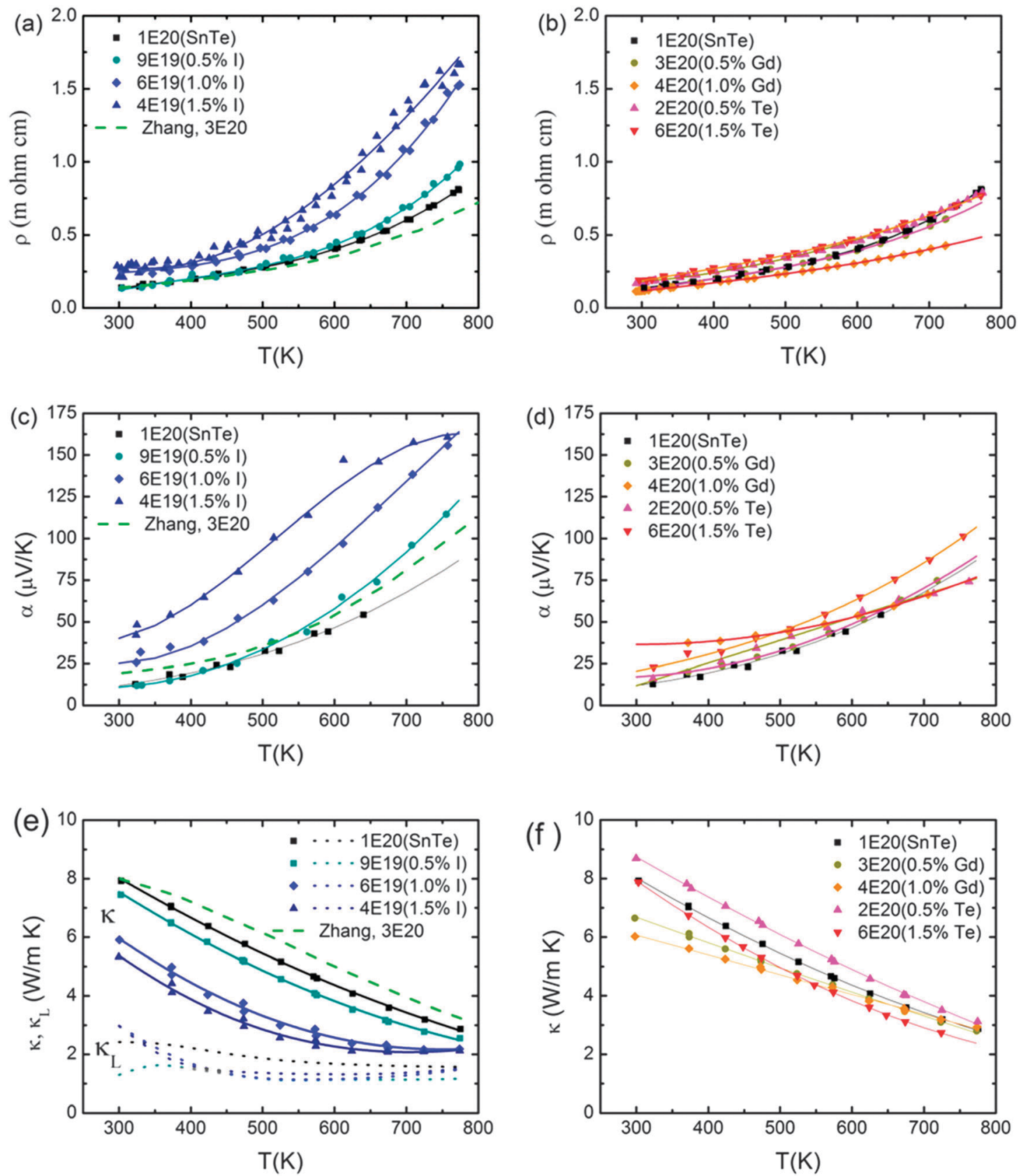

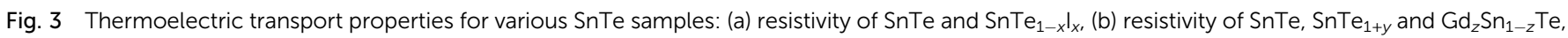
(c) Seebeck coefficients of SnTe and SnTe $e_{1-x} I_{x}$, (d) Seebeck coefficients of SnTe, SnTe $e_{1+y}$ and $\mathrm{Gd}_{z} \mathrm{Sn}_{1-z} T e$, (e) thermal conductivity and lattice thermal conductivity of SnTe and $\mathrm{SnTe}_{1-x} \mathrm{I}_{x}$ (f) thermal conductivity of $\mathrm{SnTe}, \mathrm{SnTe}_{1+y}$ and $\mathrm{Gd}_{z} \mathrm{Sn}_{1-z} \mathrm{Te}$. Legends indicate room temperature Hall carrier concentrations and a brief description of the samples as follows: $\left.S n T e_{1-x}\right|_{x}, S_{1-x} G d_{x} T e$, and $S n T e_{1+x}$ for iodine doped (a, c, e), Gd doped (b, d, f), and excess Te (b, d, f) samples respectively. All plots show both raw experimental data (points) and polynomial fits (lines).

with the decrease of Hall carrier concentrations shown in Fig. 2. This suggests that I atoms substitute for Te and supply extra electrons which compensate for the effect of intrinsic Sn vacancies. Conversely, the p-type dopants ( $\mathrm{Gd}$ and excess Te) reduce the resistivity consistent with an increase in the carrier concentration. The Seebeck coefficient of these samples, unlike in the I doping case, shows an increase with an increase in the doping level at room temperature-a direct consequence of the two-band behavior described in Fig. 1. Furthermore, the most heavily doped sample $\left(6 \times 10^{20} \mathrm{~cm}^{-3}\right)$ also has nearly the highest Seebeck coefficient at room temperature, but it does not increase as much with temperature as samples with lower doping levels.

The total thermal conductivity and the calculated lattice thermal conductivity of $\mathrm{SnTe}_{1-x} \mathrm{I}_{x}$ samples are shown in Fig. $3 \mathrm{e}$ and $\mathrm{f}$. The total thermal conductivity of the undoped SnTe decreases with temperature, reaching $2.3-3.0 \mathrm{~W} \mathrm{~m}^{-1} \mathrm{~K}^{-1}$ at $773 \mathrm{~K}$. The thermal conductivities of all the I-doped $\mathrm{SnTe}_{1-x} \mathrm{I}_{x}$ 

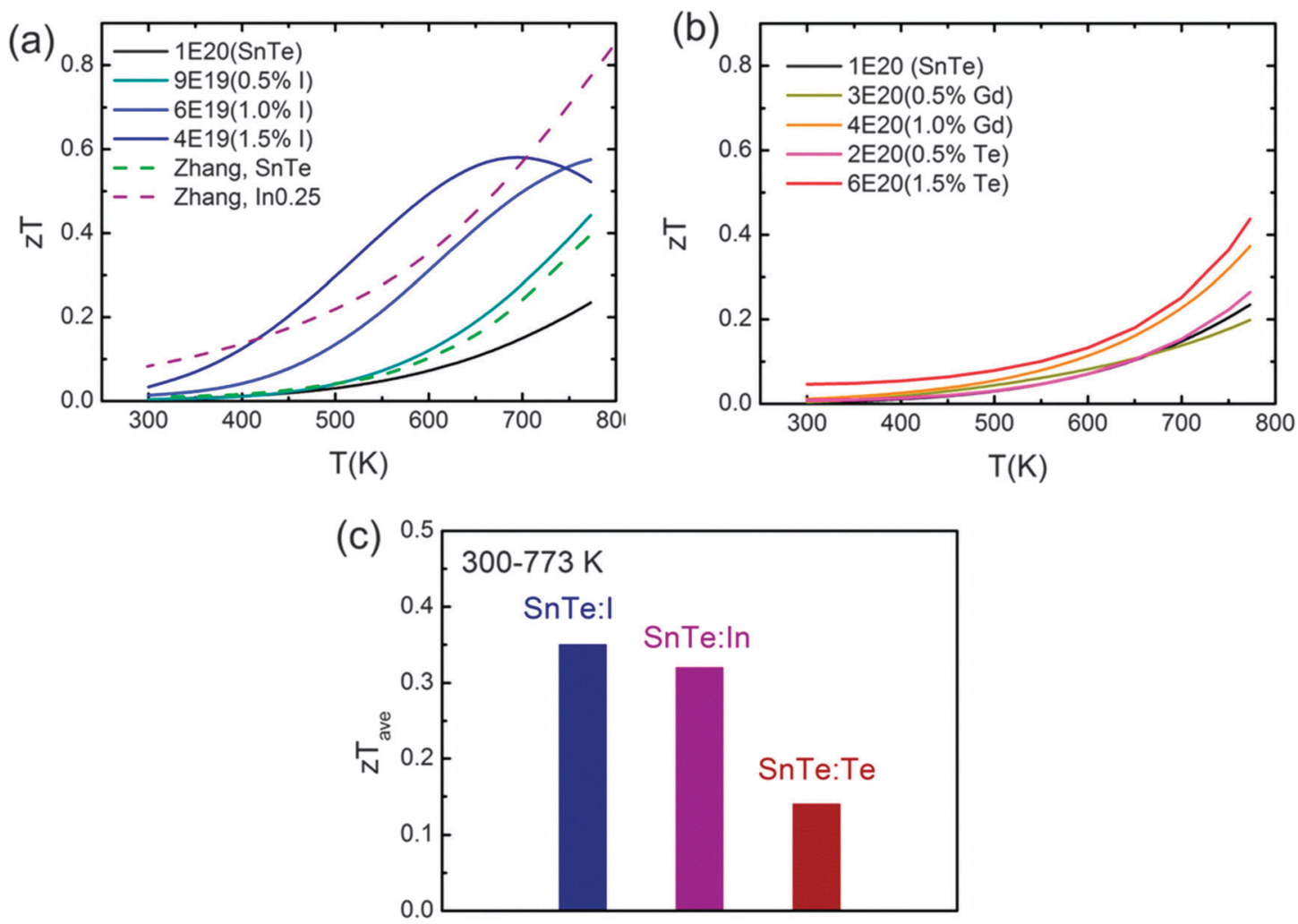

Fig. 4 (a) zT of $\left.\mathrm{SnTe}_{1-x}\right|_{x}$ as a function of temperature, data (dashed lines) are obtained from the literature. ${ }^{17}$ (b) $z T$ of $\mathrm{SnTe}_{1+y}$ and $\mathrm{Gd}_{z} \mathrm{Sn}_{1-z} \mathrm{Te}_{\mathrm{as}}$ a function of temperature. (c) The average zT between 300 and $773 \mathrm{~K}$ for optimum doped samples, data for SnTe:In are obtained from the literature. ${ }^{17}$ zT estimates are obtained from polynomial fits of transport data in Fig. 3.

are lower than that of undoped SnTe, which comes from the reduction of electronic thermal conductivity as a result of a decrease in the hole concentration. The lattice thermal conductivity, $\kappa_{\mathrm{L}}$, is calculated by subtracting the electronic contribution $\left(\kappa_{\mathrm{e}}=L T / \rho\right)$ from the total thermal conductivity, where $L$ is the Lorenz number that was estimated from a two-band model $\left(L=\left(L_{\mathrm{L}} \sigma_{\mathrm{L}} T+L_{\Sigma} \sigma_{\Sigma} T+\kappa_{\text {bipolar }}\right) /\left(\sigma_{\mathrm{L}} T+\sigma_{\Sigma} T\right)\right.$, where $\kappa_{\text {bipolar, } \mathrm{L}-\Sigma}=$ $\left.T\left(\sigma_{\mathrm{L}} \alpha_{\mathrm{L}}^{2}+\sigma_{\Sigma} \alpha_{\Sigma}^{2}-\left(\sigma_{\mathrm{L}} \alpha_{\mathrm{L}}+\sigma_{\Sigma} \alpha_{\Sigma}\right)^{2} /\left(\sigma_{\mathrm{L}}+\sigma_{\Sigma}\right)\right)\right)$, which only includes bipolar effects within the two valence bands-electron-hole bipolar effects (most commonly thought of) are not considered in this Lorenz number calculation. The lattice thermal conductivity of all the I-doped $\mathrm{SnTe}_{1-x} \mathrm{I}_{x}$ samples decreased with temperature, and then increased when the temperature is over $600 \mathrm{~K}$. This suggests that electron-hole bipolar effects occur in I-doped $\mathrm{SnTe}_{1-x} \mathrm{I}_{x}$ samples with lower carrier concentrations at high temperature. $\kappa_{\mathrm{L}}$ is not shown for Te and Gd doped samples, which were shown to be additionally complex due to large contributions from the $\Sigma$ band; instead the estimates are included in the $\mathrm{ESI}^{\dagger}$ (Fig. S3).

Full optimization of SnTe yields a higher zT (average and peak) for samples doped with iodine. The thermoelectric figure of merit, zT, is shown as a function of temperature in Fig. 4 for iodine-, Gd-, and Te-rich samples along with results of Zhang et al. for an undoped and an In doped sample. ${ }^{17}$ We show that the undoped SnTe $\left(n_{\mathrm{H}}=1.1 \times 10^{20} \mathrm{~cm}^{-3}\right)$ shows low zT values over the measured temperature range yielding a maximum of 0.23 at $773 \mathrm{~K}$. This is lower than the reported zT value $(0.39)$ of
SnTe with a higher carrier concentration $\left(n_{\mathrm{H}}=2 \times 10^{20} \mathrm{~cm}^{-3}\right)$ at the same temperature of Zhang et al. (as shown in Fig. 4a). From Fig. 4a, we can see that zT values increased with the donor I-dopant and a peak zT value of 0.6 was obtained for $\mathrm{SnTe}_{0.985} \mathrm{I}_{0.015}$ at $700 \mathrm{~K}$, corresponding to an optimum doping level of around $4 \times 10^{19} \mathrm{~cm}^{-3}$, which was the lowest attainable with iodine doping that did not lead to hysteretic behavior in the transport properties (see Fig. S5a, ESI $\dagger$ ). zT values of 0.45-0.6 were obtained for several samples with room temperature $n_{\mathrm{H}}$ of $4.0-6.1 \times 10^{19} \mathrm{~cm}^{-3}$. This means that decreasing the carrier concentration is a valid approach to optimize zT of SnTe by doping with iodine.

Alternatively, zT values also increased with the acceptor Te or Gd-dopant. The other peak zT value of 0.4 was obtained for the most heavily doped samples $\left(\mathrm{Gd}_{0.01} \mathrm{Sn}_{0.99} \mathrm{Te}\right.$ and $\mathrm{SnTe}_{1.015}$, $n_{\mathrm{H}}=4-6 \times 10^{20} \mathrm{~cm}^{-3}$ ) at $773 \mathrm{~K}$-about $30 \%$ lower than the iodine doped samples. Unlike the conventional single band behavior, we show that both I-doped SnTe and Gd-doped SnTe have higher zT values than that of stoichiometric SnTe. Fig. 4c shows the average zT value $\left(\overline{\mathrm{zT}}=\frac{\int_{300}^{773} \mathrm{zTd} T}{773-300}\right)$ of I-doped and Te-rich SnTe samples along with results of Zhang et al. obtained for $\mathrm{In}_{0.0025} \mathrm{Sn}_{0.9975} \mathrm{Te}$ samples over the temperature range of $300-773 \mathrm{~K}$. $\mathrm{SnTe}_{1.015}$ shows an average zT of 0.15 , but the average zT value of best I-doped SnTe (0.35) is about the same as the best In-doped sample (0.32) which contains 
resonant states. We believe that carrier concentration optimization will prove to be useful for $\mathrm{zT}$ enhancement in SnTe in the moderate temperature regime, without resonant impurities.

The Seebeck coefficient as a function of the Hall carrier concentration (Pisarenko plot) is shown in Fig. 1a for $\mathrm{SnTe}_{1-x} \mathrm{I}_{x}$, $\mathrm{SnTe}_{1+y}$, and $\mathrm{Gd}_{z} \mathrm{Sn}_{1-z} \mathrm{Te}$ at $300 \mathrm{~K}$ along with reported results of Brebrick and Rogers et al. ${ }^{6,9}$ The plot shows a unique, nonmonotonic $n_{\mathrm{H}}$ dependence brought about by the two interacting valence bands. For a carrier concentration of $1-2 \times 10^{20} \mathrm{~cm}^{-3}$, the Seebeck coefficient shows a minimum value of about 5-10 $\mu \mathrm{V} \mathrm{K}^{-1}$. The Seebeck coefficient then increases to a maximum of about $30 \mu \mathrm{V} \mathrm{K}^{-1}$ at a carrier concentration of $6-8 \times 10^{20} \mathrm{~cm}^{-3}$. Fig. 1b shows the relationship between the carrier mobility and the Hall carrier concentration of all the samples as well as data reported by Rogers et al. at $300 \mathrm{~K} .^{9}$ The carrier mobility of stoichiometric SnTe is about $400-500 \mathrm{~cm}^{2} \mathrm{~V}^{-1} \mathrm{~s}^{-1}$ at room temperature and always decreases with an increase in the carrier concentration for all $\mathrm{SnTe}_{1-x} \mathrm{I}_{x}, \mathrm{SnTe}_{1+y}$, and $\mathrm{Gd}_{z} \mathrm{Sn}_{1-z}$ Te samples. The experimental data for both the Seebeck coefficient and mobility are fitted by a two-band model (the solid curve) using a Kane band (SKB) for the light and a parabolic band (SPB) for the heavy valence band (as described in detail in the ESI $\dagger$ ). As shown in Fig. 5, the high temperature Seebeck coefficient and carrier mobility could also be explained by the same model by allowing the valence band offset $(\Delta E)$ and band effective mass fitting parameters to change as a function of temperature. The non-monotonic behavior of the Seebeck coefficient becomes less significant at high temperature (Fig. 5a), which is probably a result of the broadening Fermi distribution and temperature dependent shifts in the band structure. By fitting experimental results we determine that the density of states effective mass $m_{\mathrm{L}}{ }^{*}$ of the light valence band is $0.14 m_{\mathrm{e}}$ for $\mathrm{SnTe}_{1-x} \mathrm{I}_{x}$ at $300 \mathrm{~K}$, and it changes with temperature roughly according to $\mathrm{d} \ln m_{\mathrm{L}}{ }^{*} / \mathrm{d} \ln T=0.55$. Similar temperature dependence has been reported in other IV-VI compounds with a similar band structure. ${ }^{1,29,38}$ Little is known about the parameters of the heavy band, and they are difficult to determine directly using experimental techniques. As a result, they were adjusted to fit the experimental Seebeck and mobility data. The density of states effective mass $m_{\mathrm{H}}{ }^{*}$ was fit to be $1.7 m_{\mathrm{e}}$ at $300 \mathrm{~K}$, and it changes with temperature according to $\mathrm{d} \ln m_{\mathrm{H}}{ }^{*} / \mathrm{d} \ln T=0.5$. The $300 \mathrm{~K}$ values are comparable to those reported by Brebrick et al. ${ }^{6}$ The valence band offset energy, $\Delta E$, between the two bands was found to be $0.4 \mathrm{eV}$ at $300 \mathrm{~K}$ according to the fitting result and decreases roughly linearly with temperature at a rate of $3.4 \times 10^{-4} \mathrm{eV} \mathrm{K}^{-1}$.

Fig. 6 shows zT as a function of Hall carrier concentration for $\mathrm{SnTe}_{1-x} \mathrm{I}_{x}, \mathrm{SnTe}_{1+y}$ and $\mathrm{Gd}_{z} \mathrm{Sn}_{1-z} \mathrm{Te}$ samples. Note that a local minimum exists in zT vs. $n_{\mathrm{H}}$ for temperatures of 300 and $600 \mathrm{~K}$, which correspond to a carrier density of $\sim 1 \times 10^{20} \mathrm{~cm}^{-3}-$ approximately the composition of stoichiometric SnTe. At a higher temperature of $773 \mathrm{~K}$, the model predicts a single maximum in $\mathrm{zT}$ as seen in most thermoelectric materials, but the peak is broadened due to the increasing influence of the second band. From Fig. 6, we can see that both the experimental and model results indicate a significant increase in zT with a decrease in the carrier concentration, yielding a maximum in the $10^{18}-10^{19} \mathrm{~cm}^{-3}$ range. In this work, the solubility of $\mathrm{I}$ in $\operatorname{SnTe}_{1-x} \mathrm{I}_{x}\left(x=0.015, n_{\mathrm{H}} \sim 4 \times 10^{19} \mathrm{~cm}^{-3}\right)$ has limited us from achieving the optimized $n_{\mathrm{H}}$ to obtain the predicted maximum zT (which requires $n_{\mathrm{H}} \sim 8 \times 10^{18} \mathrm{~cm}^{-3}$ ). While we do expect bipolar effects to begin to play a role at low doping levels, which is not accounted for in this model, the conclusions remain that optimizing the SnTe carrier concentration towards the light band results in a significant improvement.

While both SnTe and PbTe are group IV-VI materials having the same crystal structure and similar electronic band structures, their thermoelectric performance and optimization strategies are quite different. At first, one might write off SnTe due to its large intrinsic defect concentration and higher lattice thermal conductivity when compared to PbTe. However, this work suggests that it does in fact give a reasonable zT when optimizing towards the low carrier concentration, light band over the poorer heavy band; this is achieved by doping with iodine. While valley degeneracy and band convergence play a crucial role in the high zT for PbTe (more than 1.5 at $T \sim 800 \mathrm{~K})$, the larger band offset in SnTe (0.3 eV for SnTe $v s$. $0.1 \mathrm{eV}$ for PbTe at $300 \mathrm{~K})$ makes convergence unattainable in SnTe
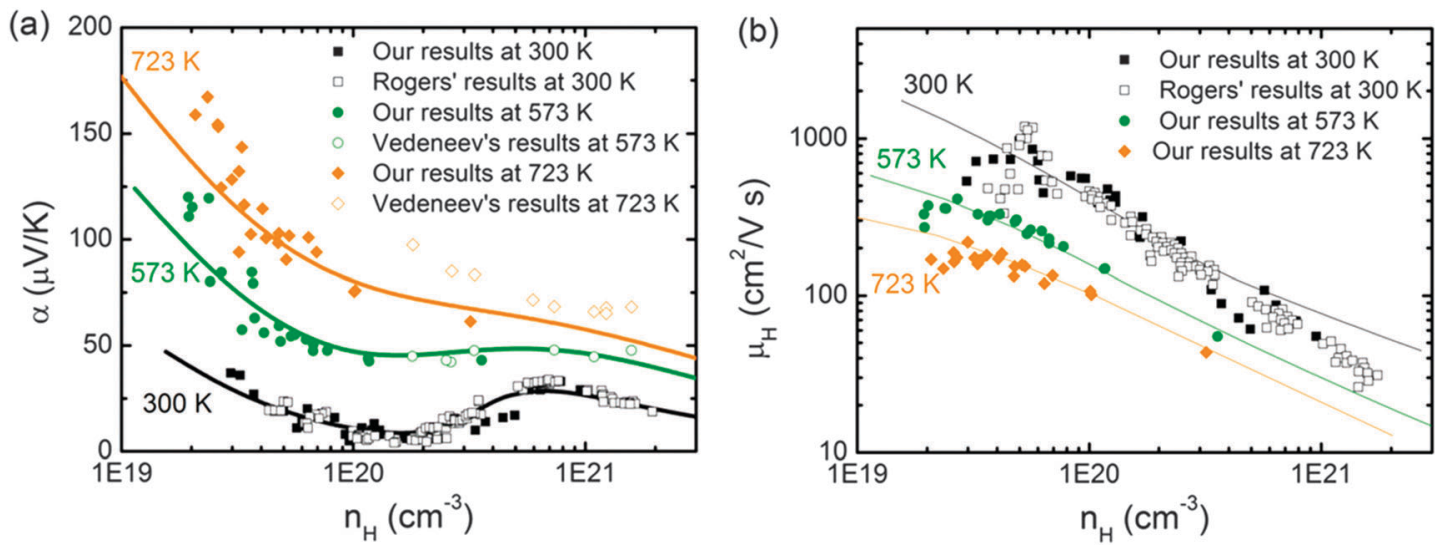

Fig. 5 (a) Seebeck coefficient and (b) Hall mobility as a function of Hall carrier concentration at different temperatures. Solid symbols represent our experimental results, open symbols correspond to literature data (Vedeneev ${ }^{7}$ and Rogers ${ }^{9}$ ). Each is presented at three temperatures: 300,573 , and $723 \mathrm{~K}$ which are given by squares, circles, and diamonds respectively. Solid curves represent the results of the two-band model. 


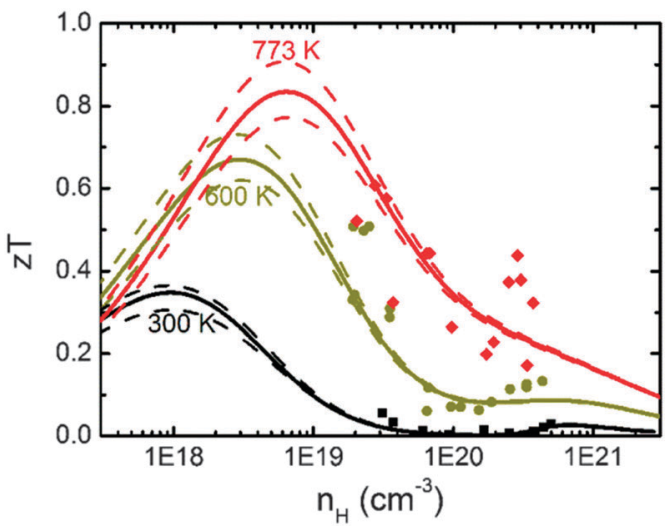

Fig. $6 z T$ as a function of Hall carrier concentration for $S n T e_{1-x} l_{x}, S_{n T e} e_{1+y}$ and $\mathrm{Gd}_{z} \mathrm{Sn}_{1-z} \mathrm{Te}$. Solid curves are modeling results, dashed lines are uncertainty values for the model $z T$ which account for a $\pm 10 \%$ error in $\kappa_{\mathrm{L}}$. The calculated lattice thermal conductivities of $2.5 \pm 10 \% \mathrm{~W} \mathrm{~m}^{-1} \mathrm{~K}^{-1}$ (300 K), $1.23 \pm 10 \% \mathrm{~W} \mathrm{~m}^{-1} \mathrm{~K}^{-1}(600 \mathrm{~K})$ and $1.0 \pm 10 \% \mathrm{~W} \mathrm{~m}^{-1} \mathrm{~K}^{-1}(773 \mathrm{~K})$ were used in the $z T$ calculation as obtained from I-doped samples (Fig. 3e).

for temperatures below its melting point. In addition, the expression for the thermoelectric quality factor ${ }^{29,39} B=$ $2 \kappa_{\mathrm{B}}^{2} T \hbar C_{\mathrm{l}} N_{\nu} / 3 \pi m_{\mathrm{i}}^{*} E_{\mathrm{def}}{ }^{2} \kappa_{\mathrm{L}}$ can be used to determine the quality factor of the light and heavy bands to be 0.42 and 0.27 , respectively, in SnTe at $600 \mathrm{~K}$. The light band is estimated to have nearly $50 \%$ higher quality factor than the heavy band in this system primarily due to the low band mass (and the corresponding high mobility). Coupled with a large band offset $\left(\sim 6 k_{\mathrm{B}} T\right.$ at $\left.600 \mathrm{~K}\right)$, the peak zT for SnTe occurs for a chemical potential near the light valence band edge. This is in contrast to PbTe where the heavy band is believed to have as good or better quality factor than the light band with a much smaller band offset $\left(\Delta E \sim 1.5 k_{\mathrm{B}} T\right.$ at $\left.600 \mathrm{~K}\right) .{ }^{39}$ So, while valley degeneracy and the heavy band at $\Sigma$ play an important role in $\mathrm{PbTe},{ }^{1}$ they are not viable options for improving zT in binary SnTe.

\section{Conclusions}

While undoped SnTe has a very poor thermoelectric performance, SnTe can be greatly improved through carrier density tuning. We have shown that by either increasing or decreasing the carrier concentration, zT can be improved relative to naturally synthesized, nominally undoped SnTe. A peak zT value of 0.6 is obtained for the $\mathrm{SnTe}_{0.985} \mathrm{I}_{0.015}$ sample with a lower carrier concentration of $4 \times 10^{19} \mathrm{~cm}^{-3}$, which is about $50 \%$ higher than the other peak zT value of 0.4 for $\mathrm{SnTe}_{1.015}$ with a higher carrier concentration of $p_{\mathrm{H}}=6 \times 10^{20} \mathrm{~cm}^{-3}$. Transport property models predict a higher $\mathrm{zT}$ if the carrier concentration could be reduced further to $1 \times$ $10^{19} \mathrm{~cm}^{-3}$. Different from In-doped SnTe that alters the host band structure, this work revealed the inherent merit of SnTe thermoelectric materials. It is worth noting that an average zT of 0.35 was obtained for light band dominated $\mathrm{SnTe}_{0.985} \mathrm{I}_{0.015}(300-773 \mathrm{~K})$; this is nearly the same as In-doped SnTe (0.32) with resonant states averaged over the same temperature range. With further band engineering SnTe may become an efficient lead-free alternative of lead chalcogenide thermoelectric materials.

\section{Acknowledgements}

The authors would like to acknowledge funding from the ITMO University in Saint Petersburg Russia. GJS and ZMG would like to acknowledge funding from The Materials Project, which is supported by Department of Energy's Basic Energy Sciences program under Grant No. EDCBEE, DOE Contract DE-AC02$05 \mathrm{CH} 11231$.

\section{References}

1 Y. Pei, X. Shi, A. LaLonde, H. Wang, L. Chen and G. J. Snyder, Nature, 2011, 473, 66-69.

2 H. Wang, Y. Pei, A. D. LaLonde and G. J. Snyder, Adv. Mater., 2011, 23, 1366-1370.

3 K. F. Hsu, S. Loo, F. Guo, W. Chen, J. S. Dyck, C. Uher, T. Hogan, E. Polychroniadis and M. G. Kanatzidis, Science, 2004, 303, 818-821.

4 I. U. r. I. Ravich, B. A. e. Efimova and I. A. Smirnov, Semiconducting lead chalcogenides, Plenum Publishing Corporation, 1970.

5 F. Herman, R. L. Kortum, I. B. Ortenburger and J. P. van Dyke, J. Phys., Colloq., 1968, 29, C4-62-C4-77.

6 R. F. Brebrick and A. J. Strauss, Phys. Rev., 1963, 131, 104.

7 V. P. Vedeneev, S. P. Krivoruchko and E. P. Sabo, Semiconductors, 1998, 32, 241-244.

8 R. L. Bernick and L. Kleinman, Solid State Commun., 1970, 8, 569-575.

9 L. M. Rogers, J. Phys. D: Appl. Phys., 1968, 1, 845-852.

10 S. Santhanam and A. Chaudhuri, Mater. Res. Bull., 1981, 16, 911-917.

11 P. Gonzalez, J. Agapito and D. Pardo, J. Phys. C: Solid State Phys., 1986, 19, 899-907.

12 A. N. Veis and Y. I. Ukhanov, Sov. Phys. Semicond., 1976, 10, 780-783.

13 D. J. Singh, Funct. Mater. Lett., 2010, 3, 223-226.

14 T. H. Hsieh, H. Lin, J. W. Liu, W. H. Duan, A. Bansil and L. Fu, Nat. Commun., 2012, 3, 982-983.

15 A. Jain, S. P. Ong, G. Hautier, W. Chen, W. D. Richards, S. Dacek, S. Cholia, D. Gunter, D. Skinner, G. Ceder and K. A. Persson, APL Mater., 2013, 1, 011002.

16 K. M. Rabe and J. D. Joannopoulos, Phys. Rev. B: Condens. Matter Mater. Phys., 1985, 32, 2302-2314.

17 Q. Zhang, B. L. Liao, Y. C. Lan, K. Lukas, W. S. Liu, K. Esfarjani, C. Opeil, D. Broido, G. Chen and Z. F. Ren, Proc. Natl. Acad. Sci. U. S. A., 2013, 110, 13261-13266.

18 G. Tan, L.-D. Zhao, F. Shi, J. W. Doak, S.-H. Lo, H. Sun, C. Wolverton, V. P. Dravid, C. Uher and M. G. Kanatzidis, J. Am. Chem. Soc., 2014, 7006-7017.

19 Y. Chen, M. D. Nielsen, Y.-B. Gao, T.-J. Zhu, X. Zhao and J. P. Heremans, Adv. Energy Mater., 2012, 2, 58-62.

20 M. K. Han, J. Androulakis, S. J. Kim and M. G. Kanatzidis, Adv. Energy Mater., 2012, 2, 157-161.

21 D. Khokhlov, L. Ryabova, A. Nicorici, V. Shklover, S. Ganichev, S. Danilov and V. Bel'kov, Appl. Phys. Lett., 2008, 93, 264103. 
22 D. R. Khokhlov, I. I. Ivanchik, S. N. Raines, D. M. Watson and J. L. Pipher, Appl. Phys. Lett., 2000, 76, 2835-2837.

23 J. V. Gumenjuk-Sichevskaya and F. F. Sizov, Semicond. Sci. Technol., 1999, 14, 1124-1131.

24 S. Y. Xu, C. Liu, N. Alidoust, M. Neupane, D. Qian, I. Belopolski, J. D. Denlinger, Y. J. Wang, H. Lin, L. A. Wray, G. Landolt, B. Slomski, J. H. Dil, A. Marcinkova, E. Morosan, Q. Gibson, R. Sankar, F. C. Chou, R. J. Cava, A. Bansil and M. Z. Hasan, Nat. Commun., 2012, 3, 1192.

25 M. Safdar, Q. S. Wang, M. Mirza, Z. X. Wang, K. Xu and J. He, Nano Lett., 2013, 13, 5344-5349.

26 K. A. Borup, E. S. Toberer, L. D. Zoltan, G. Nakatsukasa, M. Errico, J.-P. Fleurial, B. B. Iversen and G. J. Snyder, Rev. Sci. Instrum., 2012, 83, 123902.

27 S. Iwanaga, E. S. Toberer, A. LaLonde and G. J. Snyder, Rev. Sci. Instrum., 2011, 82, 063905.

28 M. Wagner, These de doctorat, Universität Wien, 2007.

29 H. Wang, Y. Z. Pei, A. D. LaLonde and G. J. Snyder, Proc. Natl. Acad. Sci. U. S. A., 2012, 109, 9705-9709.
30 D. M. Rowe, CRC Handbook of Thermoelectrics, Taylor \& Francis, 2010.

31 L. M. Rogers, Br. J. Appl. Phys., 1967, 18, 1227-1235.

32 R. F. Brebrick, J. Phys. Chem. Solids, 1963, 24, 27-36.

33 E. Rogacheva and O. Nashchekina, Phys. Status Solidi A, 2006, 203, 2856-2860.

34 T. Story, M. Gorska, A. Lusakowski, M. Arciszewska, W. Dobrowolski, E. Grodzicka, Z. Golacki and R. Galazka, Phys. Rev. Lett., 1996, 77, 3447-3450.

35 E. Trifonova and L. Karagiozov, Cryst. Res. Technol., 1983, 18, 315-320.

36 M. Moldovanova, E. Trifonova, R. Assenov and L. Karagiozov, Phys. Status Solidi A, 1980, 58, K47-K50.

37 R. Assenov, V. Moshnikov and D. Yaskov, Phys. Status Solidi A, 1985, 88, K27-K30.

38 H. Wang, E. Schechtel, Y. Pei and G. J. Snyder, Adv. Energy Mater., 2013, 3, 488-495.

39 Y. Pei, H. Wang and G. J. Snyder, Adv. Mater., 2012, 24, 6125-6135. 